\title{
Real world effectiveness of benralizumab on respiratory function and asthma control
}

\author{
Francesco Menzella, Matteo Fontana, Carla Galeone, Giulia Ghidoni, Silvia Capobelli, Patrizia Ruggiero, Chiara \\ Scelfo, Anna Simonazzi, Chiara Catellani, Francesco Livrieri, Nicola Cosimo Facciolongo \\ Pulmonology Unit, Arcispedale Santa Maria Nuova, Azienda USL di Reggio Emilia IRCCS, Reggio Emilia, Italy
}

\begin{abstract}
Background: Biological drugs have been recognized as a breakthrough in the treatment of severe refractory asthma. This retrospective real-life observational study aims to evaluate the effect of add-on benralizumab on lung function, exacerbation rate, oral corticosteroids (OCS) reduction and asthma control questionnaire (ACQ) score after 52-weeks. Methods: In this observational study, a cohort of 18 patients with severe eosinophilic asthma (SEA) according to the ERS / ATS and GINA 2020 classifications, with reference to the Pulmonology Unit of the Azienda USL - IRCCS, Reggio Emilia, Italy, were enrolled from 1 September 2019 to 31 August 2020. For each patient, the following data were collected: demographic data (age, sex, age of onset of asthma, history of smoking and atopy); comorbidity; clinical data (lung function, exacerbations, emergency room visits and hospitalizations); asthma control questionnaire (ACQ); biomarkers (blood eosinophil count and total serum IgE); asthma control drugs as high-dose inhaled corticosteroids / long-acting beta-adrenoceptor agonists (ICS / LABA), long-acting muscarinic antagonists (LAMA), leukotriene receptor antagonists (LTRA), theophylline, OCS. The benralizumab $30 \mathrm{mg}$ treatment schedule was based on the currently recommended dosing regimen.

Results: After end-of-treatment (EOT), a complete weaning of all patients from OCS was confirmed. After 26 weeks, the number of exacerbations decreased from 2.90 to 0.05 ( $\mathrm{p}<0.0001)$, hospitalizations and ACQ score decreased from 3.37 to 0.97 ( $\mathrm{p}<0.0001)$. At EOT, the number of exacerbations was unchanged, while no hospitalizations had occurred.

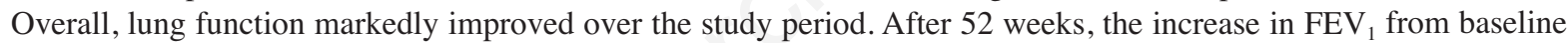
was $26,8 \%(\mathrm{p}=0.0002)$. The subset of patients with nasal polyposis (NP) had an increase of nearly $50 \%(1008 \mathrm{ml})$ and patients with blood eosinophils count (BEC) greater than 500 cells / $\mu$ l showed an increase of $68 \%$ (1081 ml) in FEV at EOT.

Conclusions: The notable improvement in respiratory function is a significant result in this study and it is much higher than what has emerged to date. This result, together with the OCS sparing effect and the excellent clinical control of asthma, makes benralizumab a reliable and safe therapeutic option for SEA.
\end{abstract}

Key words: Severe refractory asthma, oral corticosteroids, biologics, benralizumab, lung function, exacerbations.

Correspondence: Francesco Menzella, Pneumology Unit, Arcispedale Santa Maria Nuova, Azienda USL di Reggio Emilia IRCCS, 42123 Reggio Emilia, Italy. E-mail: francesco.menzella@ausl.re.it

Conflict of interest: FM has received research grants from AstraZeneca, Novartis and Sanofi; lecture fees and advisory board fees from AstraZeneca, Boehringer Ingelheim, Chiesi, GSK, Mundipharma, Angelini, Novartis, and Sanofi. The other authors declare no conflicts of interest.

Ethical approval: The protocol was approved by the Institutional Review Board Azienda USL di Reggio Emilia - IRCCS, Arcispedale Santa Maria Nuova, Reggio Emilia, Italy. This study was performed according to Good Clinical Practice (ICH Tripartite harmonized guidelines for good clinical practice 1996; Directive 91/507. EEC, The Rules Governing Medical Products in the European Community) and in compliance with Italian laws.

Consent for publication: A written informed consent for anonymous publication of their details was obtained from the patients involved in this study. The authors are available to share these forms with the journal editorial office if requested. 


\section{Introduction}

Biological drugs have been recognized as a breakthrough in the treatment of severe refractory asthma, with very evident results in terms of reducing exacerbations, improving quality of life and reducing the use of oral corticosteroids (OCS) [1,2]. This also has an impact on the OCS-related complications with a consequent reduction in the related economic costs, thanks to the significant steroid-sparing effect of biologics [2-4]. In the field of severe eosinophilic asthma (SEA), two important pharmacological options have been available for a few years, namely mepolizumab and benralizumab, and more recently also dupilumab has become usable [5]. These are effective and safe monoclonal antibodies (mAbs), but for which there have been no head-to-head comparative studies yet. Consequently, the outcomes of real-life studies about adequate follow up on available biologics are particularly important in order to correctly prescribe these drugs. As a result of the publication of the new Food and Drug Administration (FDA) guidelines on the use of real-world data (RWD) and real-world evidence (RWE) to make regulatory decisions and monitor post-market safety, the use of RWD has become increasingly important [6]. Nowadays, it is no longer just a topic of academic discussion, but it has become a relevant issue in which choices could have a direct impact on citizens' health. The reason is the idea that real-life studies can overcome some limitations of randomized controlled trials (RCTs). This is a fundamental step to validate data obtained in more artificial environments, such as the ones from RCTs. Regarding benralizumab, several studies are currently available in real-world settings. For this reason, it is important to present long-term data on efficacy and safety of benralizumab in a real-world study of severe refractory asthma patients. This retrospective real-life observational study aims to evaluate the effect of add-on benralizumab on lung function, asthma control and quality of life (QoL) after a treatment period of 52 weeks in a group of patients with OCS-dependent SEA.

\section{Methods}

\section{Study population}

In this observational study, a cohort of patients with SEA according to the ERS / ATS and GINA 2020 classifications [7,8], with reference to the Pulmonology Unit of the Azienda USL IRCCS, Reggio Emilia, Italy, were enrolled from 1 September 2019 to 31 August 2020.

For each patient, the following data were collected: demographic data (age, sex, age of onset of asthma, history of smoking and atopy); comorbidity; clinical data (lung function, exacerbations, emergency room visits and hospitalizations); asthma control questionnaire (ACQ); biomarkers (blood eosinophil count and total serum IgE); asthma control drugs as high-dose inhaled corticosteroids / long-acting beta-adrenoceptor agonists (ICS / LABA), long-acting muscarinic antagonists (LAMA), leukotriene receptor antagonists (LTRA), theophylline, OCS. Benralizumab $30 \mathrm{mg}$ treatment schedule was based on the currently recommended dosing regimen consisting of a single subcutaneous injection given at 4-week intervals for the first three doses and every 8 weeks thereafter. The primary endpoint of this study was to describe the improvement in forced expiratory volume in $1 \mathrm{~s}\left(\mathrm{FEV}_{1}\right)$ after 26 and 52 weeks of benralizumab treatment. Secondary endpoints were: assessing the rate of exacerbations, the OCS reduction and the ACQ score before and after benralizumab treatment. A treatment period of 26 and 52 weeks was chosen to highlight not only the rapidity of benralizumab on targeting primary and secondary endpoints but also the persistence of results over an appropriate time horizon. A control group was not considered in our study because patients were followed in their real setting and all underwent the same treatment. The main purpose of our study was to make a comparison with literature data, in particular with other RWE studies. This type of studies rarely includes a control group, due to the specific design and the settings in which they are conducted. This makes the magnitude of the results less strong but still applicable to clinical practice and able to provide useful information for clinicians.

\section{Statistical analysis}

The statistical analysis was performed with Prism 8.0 statistical software package (GraphPad Software, Inc., La Jolla, CA, USA). Demographic and clinical characteristics were analyzed and compared between groups at baseline and after the follow up period at 26 and 52 weeks. Continuous variables are expressed as mean \pm standard deviation (SD), while categorical variables are expressed as absolute and percentage values. Paired $t$-test and Wilcoxon test were used to compare two groups of paired continuous variables and to compute differences between means, while ANOVA test and Friedman test were used to compare three groups of paired continuous variables; $t$-test and Mann-Whitney test were used to compare two groups of unpaired variables. Chi-square test and Fischer's exact test were used for the comparison between categorical variables. A p of less than 0.05 was considered statistically significant.

\section{Results}

\section{Demographic data and comorbidities}

In total, 18 consecutive patients with SEA according to ERS / ATS 2020 guidelines and 2020 GINA classification, followed up at the Pulmonology Unit of Azienda Sanitaria Locale di Reggio Emilia / IRCCS, Italy were enrolled in this study. In this group, asthma symptoms were uncontrolled despite maximal asthma controller therapy with high doses of ICS / LABA and OCS. At baseline, all registered subjects had a blood eosinophil count (BEC) greater than 300 cells / $\mu \mathrm{L}$ and had undergone OCS treatment for at least 6 months in the year prior to study initiation. Eleven (61.1\%) patients were male and the mean age was $54 \pm 11$ years. The inflammatory phenotypes were eosinophils only for 16 patients $(89 \%)$ and a mixed atopic and eosinophilic phenotype for 2 patients (11\%). The mean BEC was $533.5 \pm 278.4$ cells / $\mu 1$, the mean total serum $\mathrm{IgE}$ value was $364.7 \pm 661.1 \mathrm{kU} / 1$. The most common comorbidities were chronic rhinosinusitis with nasal polyps (CRSwNP) (10 patients - 56\%), gastroesophageal reflux disease (GERD) (10 patients / 55\%) and bronchiectasis (6 patients / 33\%) (Table 1).

\section{Lung function}

Overall, lung function markedly improved over the study period. At baseline, 17 out of 18 patients had positive bronchodilator responses $\left(\mathrm{FEV}_{1}\right.$ increase $\geq 12 \%$ and $\geq 200 \mathrm{~mL}$ from baseline). Twenty-six weeks after the first benralizumab dose, $\mathrm{FEV}_{1}$ increased from $1.9 \pm 0.8 \mathrm{~L}(56.7 \%)$ to $2.6 \pm 1.1 \mathrm{~L}$ $(80 \%, \mathrm{p}=0,0002)$. After 52 weeks, $\mathrm{FEV}_{1}$ was $2.7 \pm 1.1(83,4 \%$, 
$\mathrm{p}=0,034$ ) (Figure 1). The morning peak expiratory flow (PEF) value, when compared to the baseline measurement, increased from $322.3 \pm 155.3 \mathrm{~L} / \mathrm{min}$ to $406.9 \pm 182.6 \mathrm{~L} / \mathrm{min}(\mathrm{p} 0.0005)$ at 6 months and $432,9 \pm 164,1$ at 52 weeks, although the latter was not statistically significant (Table 2). The subset of patients with CRSwNP had an increase of nearly 50\% (1008 ml) in $\mathrm{FEV}_{1}$ after 52 weeks, compared with those without this comorbidity, improving by $39 \%(635 \mathrm{ml})$ (Figure 2). Stratifying patients with
BEC, the group with a cut-off greater than 500 cells/ $\mu 1$ showed a $68 \%(1081 \mathrm{ml})$ increase in $\mathrm{FEV}_{1}$ after 52 weeks compared with an improvement of $31 \%(690 \mathrm{ml})$ in the group with BEC less than 500 cells / $\mu \mathrm{l}$ (Figure 3).

\section{Asthma controller therapies and OCS}

All patients enrolled in the study were taking an ICS / LABA inhalation therapy combination, $67 \%$ of patients were taking
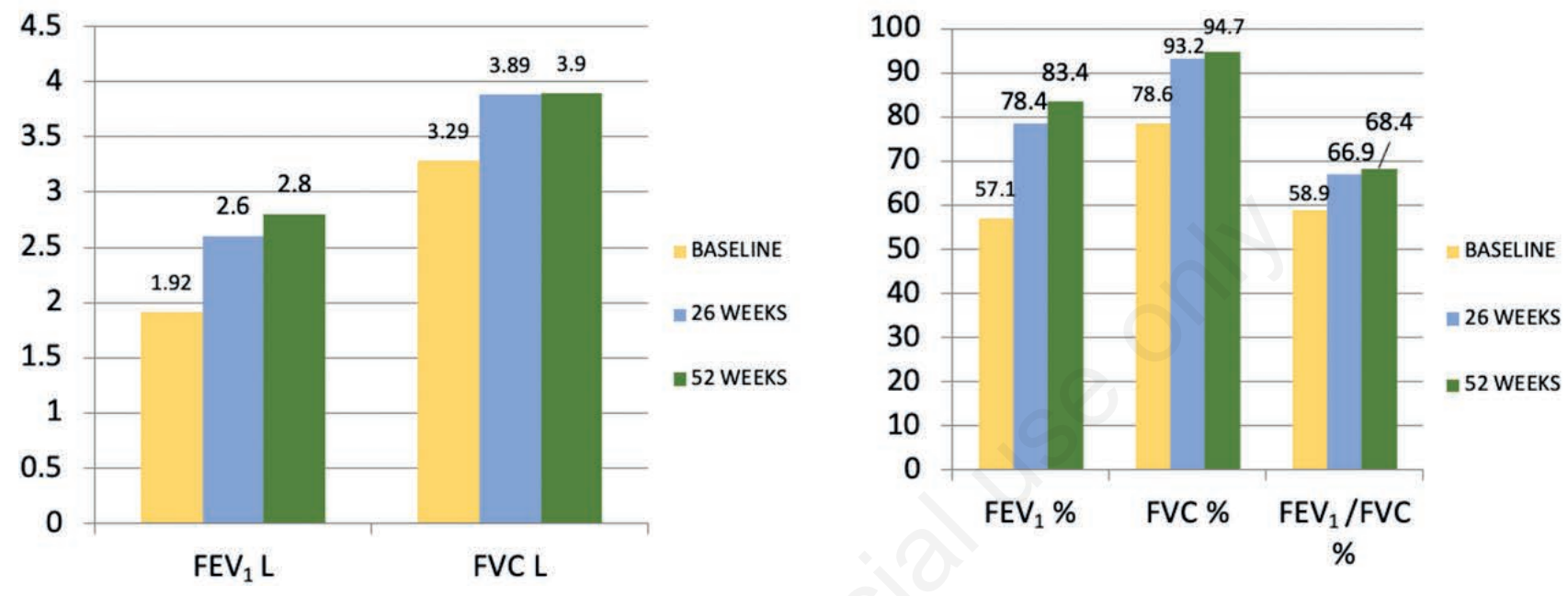

Figure 1. Lung fuction trend $(\mathrm{L} / \%)$ in the overall population.

Table 1. Demographic and clinical characteristics of patients with severe asthma.

\begin{tabular}{|c|c|}
\hline Demographic and clinical characteristics & Baseline \\
\hline Number of patients & 18 \\
\hline Age y, mean \pm SD & $54 \pm 11.7$ \\
\hline Female, n (\%) & $7(38.9)$ \\
\hline Weight Kg, mean \pm SD & $72.44 \pm 11.92$ \\
\hline History of smoking (former), n (\%) & $2(11.1)$ \\
\hline Age onset $y$, mean \pm SD & $36.3 \pm 15.84$ \\
\hline Total serum IgE KU/, mean \pm SD & $227.22 \pm 300.82$ \\
\hline Positive bronchodilator response, n (\%) & $17(94.4)$ \\
\hline Positive bronchodilator response $\%$, mean \pm SD & $16.7 \pm 8.3$ \\
\hline \multicolumn{2}{|l|}{ T2 high phenotype } \\
\hline Only eosinophilic, $\mathrm{n}(\%)$ & $16(88.9)$ \\
\hline Eosinophilic and allergic, n (\%) & $2(11.1)$ \\
\hline \multicolumn{2}{|l|}{ OCS treatment } \\
\hline Patients taking OCS at least 6 months/year n (\%) & $18(100)$ \\
\hline \multicolumn{2}{|l|}{ Comorbidities } \\
\hline CRSwNP, n (\%) & $10(55 ., 6)$ \\
\hline GERD, n (\%) & $10(55.6)$ \\
\hline Bronchiectasis, n (\%) & $6(33.3)$ \\
\hline
\end{tabular}

T2 high, type 2 high; OCS, oral corticosteroids; CRSwNP, chronic rhinosinusitis with nasal polyposis; GERD, gastroesophageal reflux disease. 
LAMA, $39 \%$ of patients were taking LTRA and $11 \%$ of patients were taking theophylline at baseline. The mean daily dose of beclomethasone was $1556 \pm 511.3 \mathrm{mcg}$. All patients had taken OCS for at least 6 months during the 12 months prior to enrollment, 6 $(30 \%)$ patients were on chronic OCS treatment for 12 months prior to benralizumab treatment. The daily dose of prednisone was $18.4 \pm$ $5.8 \mathrm{mg}$. Twenty-six weeks after the initiation of benralizumab, no patient was taking OCS daily with a general reduction in the average number of drugs taken; the daily dose of beclomethasone was $1194 \pm 546.1 \mathrm{mcg}(\mathrm{p}=0.0078)$ (Table 2). All the average doses of concomitant medications decreased. After 52 weeks, complete weaning of all patients from OCS was confirmed, the daily dose of beclomethasone was further decreased to $989.9 \pm 386.4(\mathrm{p}=0.031)$.

\section{Asthma control parameters and blood eosinophils}

At baseline, the mean number of annual exacerbation rate
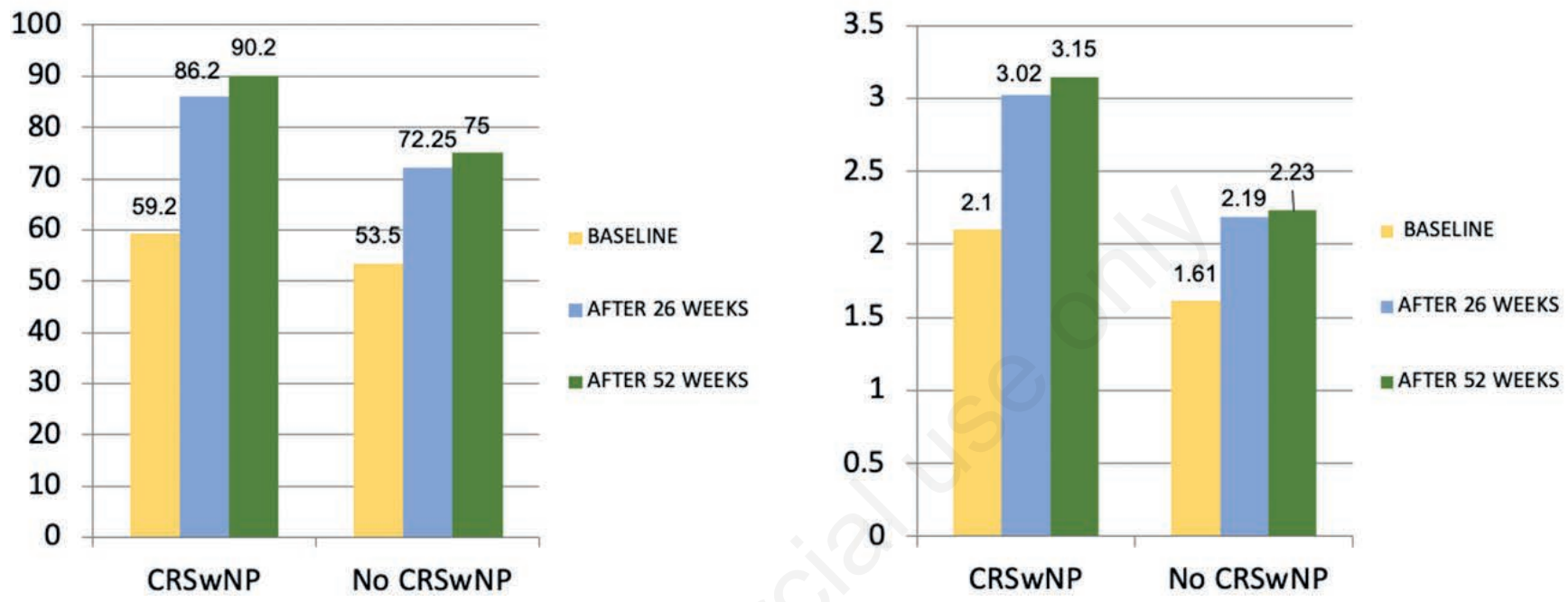

Figure 2. $\mathrm{FEV}_{1}(\% / \mathrm{L})$ improvement in the CRSwNP subgroup. CRSwNP, chronic rhinosinusitis with nasal polyps.

Table 2. Variations of clinical outcomes between baseline and after 26 and 52 weeks after benralizumab therapy.

\begin{tabular}{|c|c|c|c|c|c|}
\hline & Baseline & 26 weeks & 52 weeks & Change from baseline & $\mathbf{p}$ \\
\hline Eosinophils & $530 \pm 294.1$ & $0.02 \pm 0.04$ & $0.02 \pm 0.04$ & $-530 \pm 294.1$ & $<0.0001$ \\
\hline ACQ & $3.3 \pm 0.6$ & $0.8 \pm 0.5^{*}$ & $0.5 \pm 0.3^{*}$ & $-2.8 \pm 0.7$ & $<0.0001$ \\
\hline Hospitalizations & $0.1 \pm 0.3$ & $0 \pm 0$ & $0 \pm 0$ & $-0.1 \pm 0.3$ & 0.13 \\
\hline Exacerbations & $2.8 \pm 0.7$ & $0.05 \pm 0.2$ & $0.05 \pm 0.2$ & $-2.78 \pm 0.7$ & $<0.0001$ \\
\hline PEF & $322.3 \pm 155.3$ & $406.9 \pm 182.6$ & $432.9 \pm 164.1$ & $+110.6 \pm 116.3$ & 0.0001 \\
\hline $\mathrm{FEV}_{1} \%$ & $56.7 \pm 12.9$ & $80 \pm 23.6 \#$ & $83.4 \pm 25.4^{\sharp}$ & $+26.8 \pm 23.9$ & 0.0002 \\
\hline $\mathrm{FEV}_{1} \mathrm{~L}$ & $1.9 \pm 0.8$ & $2.6 \pm 1.1^{\circ}$ & $2.7 \pm 1.1^{\circ}$ & $+0.8 \pm 0.7$ & $<0.0001$ \\
\hline FVC $\%$ & $77.5 \pm 7.6$ & $93.4 \pm 18.7$ & $94.7 \pm 21$ & $+17.2 \pm 19.5$ & 0.0003 \\
\hline FVC L & $3.2 \pm 0.9$ & $3.9 \pm 1.2$ & $3.9 \pm 1.3$ & $+0.7 \pm 0.8$ & 0.0007 \\
\hline $\mathrm{FEV}_{1} / \mathrm{FVC}$ & $59.4 \pm 13.3$ & $68.3 \pm 13.8$ & $68.4 \pm 11.5$ & $+8.9 \pm 10.4$ & 0.039 \\
\hline ICS (mcg) & $1556 \pm 511.3$ & $1194 \pm 546.1^{\S}$ & $989.9 \pm 386.4^{\S}$ & $-566.7 \pm 470.3$ & $<0.0001$ \\
\hline OCS (mg) & $18.4 \pm 15.8$ & $0 \pm 0$ & $0 \pm 0$ & $-18.4 \pm 15.8$ & 0.0067 \\
\hline LAMA & $12(67)$ & $7(39)$ & $5(28)$ & $-7(58)$ & 0.054 \\
\hline Anti-leukotriens & $7(39)$ & $4(22)$ & $4(22)$ & $-3(43)$ & 0.43 \\
\hline Theophylline & $2(11)$ & $1(5)$ & $1(5)$ & $-1(50)$ & 0.76 \\
\hline GINA step 5 & $14(78)$ & $8(44)$ & $6(33)$ & $-8(57)$ & 0.02 \\
\hline
\end{tabular}

ACQ, asthma control questionnaire; PEF, peak expiratory flow; FEV 1 , forced expiratory volume in 1 s; FVC, forced vital capacity; ICS, inhaled corticosteroids; OCS, oral corticosteroids; LAMA, long-acting muscarinic antagonists; statistically significant differences between data at 26 and 52 weeks are indicated: ${ }^{*} 0.0005,{ }^{\circledR} 0.026,{ }^{\circ} 0.034,{ }^{8} 0.031$. 
(AER) was 2.8 \pm 0.7 , Emergency Department (ED) visits and hospitalizations were $0.1 \pm 0.3$ and the ACQ score was $3.3 \pm 0.6$. After 26 weeks of benralizumab treatment, the number of exacerbations decreased to $0.05 \pm 0.2(p<0.0001)$, there were no more ED visits and hospitalizations and ACQ scores decreased to $0.8 \pm 0.5(\mathrm{p}<0.0001)$ (Table 2). At 52 weeks of follow up, the number of exacerbations was unchanged, while no hospitalization had occurred. At the end-of-treatment (EOT), the ACQ score dropped further $(0.5 \pm 0.3, \mathrm{p}<0.005)$. Twenty-six weeks after the first dose of benralizumab, the BEC significantly decreased from $533.5 \pm 278.4$ cells $/ \mu 1$ to $0.02 \pm 0.04$ cells $/ \mu 1(p<0.0001)$. After 52 weeks this value was unmodified. Analyzing the subsets of patients with CRSwNP and without CRSwNP, the first had a BEC of $599 \pm 362.9$ cells $/ \mu$ while the second of $443.8 \pm 159.3$ cells / $\mu 1$ at baseline. At 52 weeks, the reduction in both subgroups was considerable and statistically significant $(\mathrm{p}<0.0001)$.

\section{Safety profile}

No mild or severe side effects were observed throughout the treatment period.

\section{Discussion}

In the context of severe "Type-2 (T2) high" and eosinophilic asthma it is not easy to define which is the most effective biologic, since comparative head-to-head studies have not been conducted yet. Evidence of efficacy and safety is good for all currently available mAbs, albeit with differences in respiratory function improvement and OCS sparing effect above all. The efficacy of benralizumab was demonstrated by phase 3 RCTs SIROCCO and CALIMA, which showed that this mAb significantly reduced the annual rate of exacerbations, improved $\mathrm{FEV}_{1}$ and reduced asthma symptoms $[9,10]$. Another RCT, the ZONDA study, showed that benralizumab had also a good OCS sparing profile [11]. In a post hoc analysis of the SIROCCO and CALIMA studies, the response to benralizumab was assessed on the basis of fixed airflow

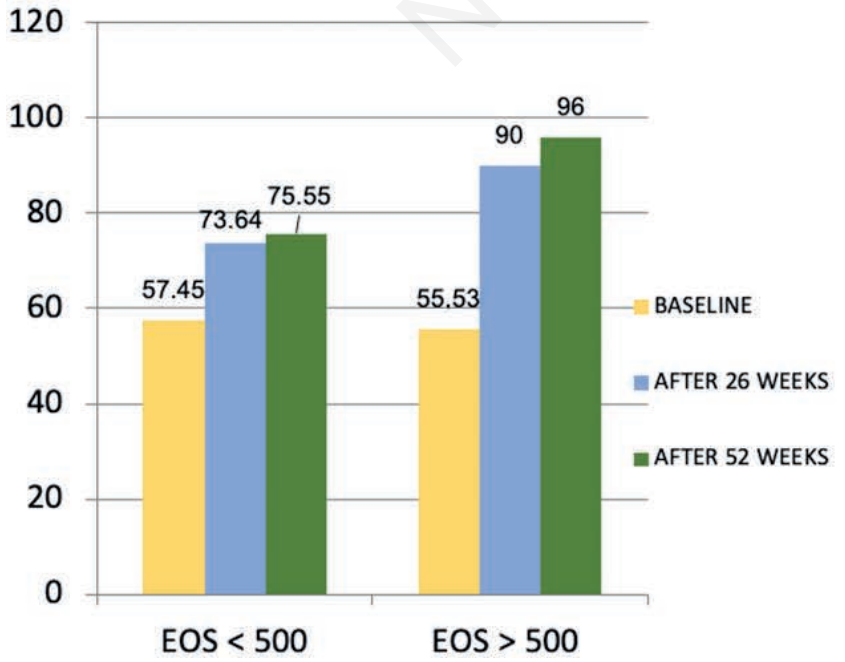

obstruction (FAO) status, with a prevalence of 63\% (935/1493 patients). Benralizumab improved the rate of exacerbations, lung function and quality of life to a greater extent for FAO + patients than for FAO - patients [12]. RWE studies are usally conducted on more heterogeneous patients cohorts than RCTs; they also confirmed (sometimes with better results) the efficacy of benralizumab in asthma control, improvement of respiratory function and OCS sparing effect. It is worth highlighting that the inclusion and exclusion criteria are less restrictive in real-world studies than in RCTs; in fact, real-world studies only require the diagnosis of SEA and the presence of the prescription criteria for benralizumab. A recent Italian real-life study confirmed the efficacy of benralizumab in terms of improving FEV $_{1}$ and PEF as well as weaning from OCS, with results superior to those of RCTs [13]. In another multicenter real-life study in patients with SEA and nasal polyposis (NP), the efficacy of benralizumab was higher in patients with NP in terms of OCS sparing effect, while it was the opposite for the respiratory function [14]. A recent multicenter real-life study on 111 atopic and non-atopic subjects with difficultto-treat eosinophilic asthma, showed that 6 months of add-on therapy with benralizumab had allowed a marked reduction in the rate of asthma exacerbation, OCS intake, daily use of short-acting 2- adrenergic agonists (SABA), asthma control test (ACT) score, asthma quality of life questionnaire score (AQLQ), respiratory function, BEC levels, blood basophil count and fractional exhaled nitric oxide (FeNO) levels. An even more interesting result was that related to the better outcomes highlighted by the subgroup of atopic patients, but only with regard to the number of exacerbations, ACT score and daily use of SABA [15]. The efficacy and rapidity of benralizumab was also confirmed by a case report on a patient with severe refractory eosinophilic asthma, reluctant to take systemic steroids, who was successfully treated with benralizumab alone during an acute attack of SEA [16].

Also in our previous RWE study, benralizumab showed great efficacy, not only in terms of exacerbation rate reduction but above all on the improvement in respiratory function and OCS sparing effect after 26 weeks of treatment. These results were even higher in the subgroup of patients with NP and BEC $>500$ cells/ $\mu 1$ [17].

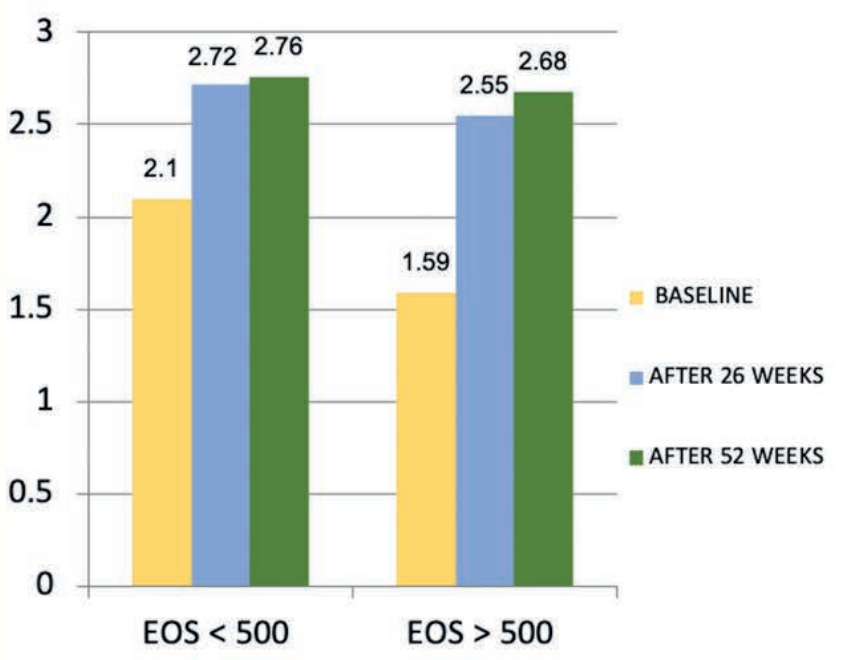

Figure $3 . \mathrm{FEV}_{1}(\% / \mathrm{L})$ improvement according to blood eosinophil count $>500$ cells $/ \mu \mathrm{l}$. 
There is evidence confirming that NP is a useful clinical factor or phenotype in patients with CRS that may enhance therapeutic response to benralizumab [18]. The reason is a strong relationship between cellular and molecular mechanisms underlying type 2 inflammation in asthma and NP, especially in eosinophilic inflammation. Furthermore, patients with SEA and concomitant NP often have higher BEC, worse respiratory function at baseline and a higher average OCS daily dosage [19].

At baseline, the 18 patients enrolled in our study were taking OCS continuously or nearly continuously. In our study, the average daily dose of prednisone at baseline $(18.4 \mathrm{mg} / \mathrm{die})$ was higher than in patients enrolled in pivotal studies on benralizumab as SIROCCO (15.2 mg/day in $18 \%$ of patients) [9] and on mepolizumab as MENSA (12.6 mg/day in 27\% of patients) [20]. This dose is also higher than the average daily dose of patients enrolled in the ZONDA study (10 mg/day) [11] on benralizumab and in the SIRIUS study (12.5 mg/day) [21] on mepolizumab. The add-on therapy with the anti-eosinophil biologic benralizumab allowed to completely withdraw the OCS in all the patients examined already after 26 weeks, confirming the weaning after 52 weeks. This result was achieved despite the high average daily dose of OCS in the patients enrolled in our study. This result confirmed that the antibody-dependent cell-mediated cytotoxicity (ADCC) on effector cells (eosinophils, basophils and type 2 innate lymphoid cells (ILC2) caused by benralizumab [2] and the complete inhibition of IL-5 activity have an extremely profound anti-inflammatory activity, which then led to a significant improvement of asthma control.

The improvement in lung function $\left(\mathrm{FEV}_{1}+842 \mathrm{ml}\right)$ observed in our patients was also remarkably high, superior to the data of RWE and RCTs available in the literature so far [9,12-14,22]. The best result was obtained in the subset of patients with BEC $>500$ cells $/ \mu \mathrm{l}\left(\mathrm{FEV}_{1}+1081 \mathrm{ml}\right)$ and those with concomitant NP $\left(\mathrm{FEV}_{1}\right.$ $+1008 \mathrm{ml})$.

Benralizumab has also confirmed its efficacy from a clinical point of view. At EOT there were no hospitalization and exacerbation and the ACQ score improved significantly after 26 weeks with the best result after 52 weeks $(-2.8$ points, $\mathrm{p}<0.0001)$. These results, together with the previous ones, confirm that the benefits of benralizumab are certainly rapid but progressively increase over time, especially in patients with eosinophilic asthma who are refractory to OCS, with CRSwNP or a combination of this clinical characteristics.

Based on the outcomes described above, the subgroup of patients with BEC $>500$ cells $/ \mu$ l and NP can be defined as "super responders" (defined as zero exacerbations, notable improvement in respiratory function and no OCS therapy for asthma) similarly to what emerged from other recent RWE studies [23].

As for eosinophils, similarly to what is widely known, an almost complete depletion of these cells was observed and maintained over time in our study. Eosinophils are known to have several subtypes, most notably normodense eosinophils which are present in the parenchyma at steady state, but they also accumulate in the peribronchial and perivascular areas in T2-high asthma endotype. On the other hand, hypodense eosinophils are associated with asthmatic inflammation and are sensitive to the action of IL5 [24]. Consequently, eosinophil depletion has no impact on safety, as reservoirs of these cells are available and they are not significantly sensitive to the action of benralizumab and other antiIL-5 biologic.

The principal limitations of this study are the small number of patients enrolled and the absence of a control group, as it is a reallife retrospective study. The lack of a control group makes the magnitude of the observed results less reliable than the same data compared with a control group. However, it must be remembered that the RWE studies are often devoid of the control group.
Moreover, evaluating data generated from RWE and RTC studies will provide comprehensive insights on these therapeutic biologics and enable learnings that may be implemented in clinical practice.

\section{Conclusions}

The more important aspects of our study are the outcomes obtained in terms of significant improvement in respiratory function, which are the highest that has emerged so far in this field in literature, to our knowledge. These results, together with the OCS sparing effect and the excellent clinical control of asthma, make benralizumab an extremely reliable and safe therapeutic option in the context of severe asthma, worth to be taken into high consideration in case of patients with steroid-resistant or dependent SEA with or without CRSwNP.

$\begin{array}{ll}\text { Abbreviations } \\ \text { SEA: } & \text { severe eosinophilic asthma; } \\ \text { OCS: } & \text { oral corticosteroids; } \\ \text { mAbs: } & \text { monoclonal antibodies; } \\ \text { FDA: } & \text { food and drug administration; } \\ \text { RWD: } & \text { real-world data; } \\ \text { RWE: } & \text { real-world evidence; } \\ \text { RCTs: } & \text { randomized clinical trials; } \\ \text { QoL: } & \text { quality of life; } \\ \text { ACQ: } & \text { asthma control questionnaire; } \\ \text { ICS/LABA: } & \text { inhaled corticosteroids/ } \\ & \text { long-acting beta-adrenoceptor agonists; } \\ \text { LAMA: } & \text { long-acting muscarinic antagonists; } \\ \text { LTRA: } & \text { leukotriene receptor antagonists; } \\ \text { BEC: } & \text { blood eosinophil count; } \\ \text { FEV }: & \text { forced expiratory volume in 1 s, } \\ \text { CRSwNP: } & \text { chronic rhinosinusitis with nasal polyps; } \\ \text { GERD: } & \text { gastroesophageal reflux disease; } \\ \text { AER: } & \text { annualized exacerbation rate; } \\ \text { PEF: } & \text { peak expiratory flow; } \\ \text { ED: } & \text { emergency department; } \\ \text { T2: } & \text { high type-2 high; } \\ \text { NP: } & \text { nasal polyposis. } \\ & \end{array}$

\section{References}

1. Hearn AP, Kent BD, Jackson DJ. Biologic treatment options for severe asthma. Curr Opin Immunol 2020;66:151-60.

2. Bourdin A, Adcock I, Berger P, Bonniaud P, Chanson P, Chenivesse $C$, et al. How can we minimise the use of regular oral corticosteroids in asthma? Eur Respir Rev 2020;29:190085.

3. Canonica GW, Blasi F, Paggiaro P, Senna G, Passalacqua G, Spanevello A, et al. Oral corticosteroid sparing with biologics in severe asthma: A remark of the Severe Asthma Network in Italy (SANI). World Allergy Organ J 2020;13:100464.

4. Albers FC, Müllerová H, Gunsoy NB, Shin JY, Nelsen LM, Bradford ES, et al. IDEAL study. J Asthma 2018;55:152-60.

5. Menzella F, Ruggiero P, Ghidoni G, Fontana M, Bagnasco D, Livrieri F, et al. Anti-IL5 therapies for severe eosinophilic asthma: Literature review and practical insights. J. Asthma Allergy 2020;13:301-13.

6. U.S. Food and Drug Administration. Real-world evidence. Accessed: 30 April 2021. Available from: https://www.fda.gov/science-research/science-and-research- 
special-topics/real-world-evidence

7. Holguin F, Cardet JC, Chung KF, Diver S, Ferreira DS, Fitzpatrick A, et al. Management of severe asthma: a European Respiratory Society/American Thoracic Society guideline. Eur Respir J 2020;55:1900588.

8. Global Initiative for Asthma (GINA). 2020 GINA Main Report. Accessed: 3 April 2021. Available from: https://ginasthma.org/gina-reports

9. Bleecker ER, FitzGerald JM, Chanez P, Papi A, Weinstein SF, Barker $\mathrm{P}$, et al. Efficacy and safety of benralizumab for patients with severe asthma uncontrolled with high-dosage inhaled corticosteroids and long-acting $\beta 2$-agonists (SIROCCO): a randomised, multicentre, placebo-controlled phase 3 trial. Lancet 2016;388:2115-2127.

10. FitzGerald JM, Bleecker ER, Nair P, Korn S, Ohta K, Lommatzsch $\mathrm{M}$, et al. Benralizumab, an anti-interleukin-5 receptor $\alpha$ monoclonal antibody, as add-on treatment for patients with severe, uncontrolled, eosinophilic asthma (CALIMA): a randomised, double-blind, placebo-controlled phase 3 trial. Lancet 2016;388:2128-41.

11. Nair P, Wenzel S, Rabe KF, Bourdin A, Lugogo NL, Kuna P, et al. oral glucocorticoid-sparing effect of benralizumab in severe asthma. N Engl J Med 2017;376:2448-58.

12. Chipps BE, Hirsch I, Trudo F, Alacqua M, Zangrilli JG. Benralizumab efficacy for patients with fixed airflow obstruction and severe, uncontrolled eosinophilic asthma. Ann Allergy Asthma Immunol 2020;124:79-86.

13. Pelaia C, Busceti MT, Vatrella A, Rago GF, Crimi C, Terracciano $\mathrm{R}$, et al. Real-life rapidity of benralizumab effects in patients with severe allergic eosinophilic asthma: Assessment of blood eosinophils, symptom control, lung function and oral corticosteroid intake after the first drug dose. Pulm Pharmacol Ther 2019;58:101830.

14. Bagnasco D, Brussino L, Bonavia M, Calzolari E, Caminati M, Caruso $\mathrm{C}$, et al. Efficacy of benralizumab in severe asthma in real life and focus on nasal polyposis. Respir Med 2020;171:106080.

15. Pelaia C, Crimi C, Benfante A, Caiaffa MF, Calabrese C,
Carpagnano GE, et al. Therapeutic effects of benralizumab assessed in patients with severe eosinophilic asthma: Real-life evaluation correlated with allergic and non-allergic phenotype expression. J Asthma Allergy 2021;14:163-73.

16. Nolasco S, Campisi R, Intravaia R, Porto M, Pelaia C, Crimi N, et al. Case Report: Acute effect of benralizumab on asthma exacerbation without concomitant corticosteroid use. F1000Res 2020;9:637.

17. Menzella F, Ruggiero P, Galeone C, Scelfo C, Bagnasco D, Facciolongo N. Significant improvement in lung function and asthma control after benralizumab treatment for severe refractory eosinophilic asthma. Pulm Pharmacol Ther 2020;64:101966.

18. Bleecker ER, Wechsler ME, FitzGerald JM, Menzies-Gow A, Wu Y, Hirsch I, et al. Baseline patient factors impact on the clinical efficacy of benralizumab for severe asthma. Eur Respir J 2018;52:1800936.

19. Heffler E, Blasi F, Latorre M, Menzella F, Paggiaro P, Pelaia G, et al. The Severe Asthma Network in Italy: Findings and perspectives. J Allergy Clin Immunol Pract 2019;7:1462-8.

20. Ortega HG, Liu MC, Pavord ID, Brusselle GG, FitzGerald JM, Chetta A, et al. Mepolizumab treatment in patients with severe eosinophilic asthma. N Engl J Med 2014;371:1198-207.

21. Bel EH, Wenzel SE, Thompson PJ, Prazma CM, Keene ON, Yancey SW, et al. Oral glucocorticoid-sparing effect of mepolizumab in eosinophilic asthma. $\mathrm{N}$ Engl J Med 2014;371:1189-97.

22. Kolbeck R, Kozhich A, Koike M, Peng L, Andersson CK, Damschroder MM, et al. MEDI-563, a humanized anti-IL-5 receptor alpha $\mathrm{mAb}$ with enhanced antibody-dependent cellmediated cytotoxicity function. J Allergy Clin Immuno. 2010;125:1344-53.e2.

23. Kavanagh JE, Hearn AP, Dhariwal J, d'Ancona G, Douiri A, Roxas C, et al. Real-world effectiveness of benralizumab in severe eosinophilic asthma. Chest 2021;159:496-506.

24. Van Hulst G, Batugedara HM, Jorssen J, Louis R, Bureau F, Desmet CJ. Eosinophil diversity in asthma. Biochem Pharmacol 2020;179:113963.

Received for publication: 20 May 2021. Accepted for publication: 22 July 2021.

This work is licensed under a Creative Commons Attribution-NonCommercial 4.0 International License (CC BY-NC 4.0).

(C) Copyright: the Author(s), 2021

Licensee PAGEPress, Italy

Multidisciplinary Respiratory Medicine 2021; 16:785

doi:10.4081/mrm.2021.785 